two cases in the placebo vaccine cohort, and 21 cases in the unvaccinated reference cohort suggesting that the vaccine efficacy translates into efficacy against cervical cancer. The passive follow-up continues and new cases emerging in future will be monitored by redoing linkage with the population-based cancer register at specific time intervals in the future, which will effectively add up person years to our follow-up study. In conclusion, valid comparisons between the vaccine and placebo recipients (excluding cross-vaccinated placebo vaccine recipients) and the reference cohort not exposed to intervention are feasible, and will be critical to define more definitively the long-term protection provided by HPV vaccination against the hard endpoints.

\section{3-S2.03 THE SPECTRUM OF GENITAL HPV INFECTION AMONG MEN ATTENDING A SWEDISH STI CLINIC: HPV TYPING AND CLINICAL PRESENTATION}

doi:10.1136/sextrans-2011-050109.111

${ }^{1}$ A Wikström, ${ }^{1} \mathrm{M}$ A Hedblad, ${ }^{2}$ S Syrjänen. ${ }^{1}$ Karolinska University Hospital, Stockholm, Sweden; ${ }^{2}$ University of Turku, Turku, Finland

Background Some Swedish studies on HPV typing in men exist. Most earlier studies have used less sensitive HPV typing techniques. The purpose of this study is to see if the HPV types in genital HPV associated lesions have changed since the $80 \mathrm{ies}$, and to describe the lesions in detail.

Methods Between 2004 and 2007, male patients attending the STI clinic of Karolinska Hospital with genital HPV induced lesions planned for surgery, were asked to participate in the study. All men exhibited multiple lesions-men with solitary lesions were excluded. Two clinically identical lesions on the same genital site, were collected by punch biopsy or by scissor excision. One sample was put in formalin for histopathological routine preparation, and the other sample was frozen in $-70^{\circ} \mathrm{C}$ for PCR analysis using a highly sensitive nested PCR technique, detecting 24 different HPV types. The macroscopic morphology of the lesions was classified in acuminate, papular, macular and seborrhoeic keratosis like. The colour of the lesions and the location were recorded. Data on previous therapy and how long time the patients had been afflicted with genital symptoms/and or warts was also noted.

Results Totally 303 men were included in the study. Of these, 47 men $(16 \%)$ exhibited lesions of PIN and have been described previously. The remaining 256 men had benign lesions and are described here. Acuminate lesions dominated, occurring in 106 (41\%) of the men, followed by papular lesions found in 88 (34\%) men. The penile shaft, the pubic area and the foreskin were the most common locations for the HPV induced lesions, afflicted in 36\%, 29\% and 25\% of the men, respectively. Pink and brown were the dominating colours of the leions. HPV was detected in 233 (91\%) lesions. Low risk HPV types only, were found in $75 \%$ of the lesions. On the other hand, $7 \%$ of the lesions contained only high risk HPV types, and 9\% had a mix of low- and high risk HPV types. Multiple HPV types were found in $13 \%$. HPV 6 was the most common HPV type $(70 \%$ of the lesions were positive for HPV 6 only). Duration of genital symptoms and/or warts was mean 24 months and 211 of the men had previously been treated.

Conclusion Using a highly sensitive PCR technique, a high HPV detection rate of $91 \%$ was found. As in earlier studies, HPV 6 was most common, but also other HPV types including high-risk types were detected. As expected, most of the benign lesions were acuminate, but the morphology as well as the genital location varied.

\section{3-S2.04 CERVICAL ECTOPY IS NOT ASSOCIATED WITH ACQUISITION OF HPV INFECTION}

doi:10.1136/sextrans-2011-050109.112

L Y Hwang, J Lieberman, Y Ma, A B Moscicki. University of California, San Francisco, USA

Background HPV infection and cervical cancer are often found at the cervical transformation zone. High rates of HPV in adolescents have been attributed to their greater extent of "cervical ectopy", defined as areas of columnar and metaplastic epithelium visible on the ectocervix. The study aim was to examine associations between the extent of cervical ectopy and incident HPV infection in healthy adolescents.

Methods Sexually active young women were enrolled as part of a prospective HPV Natural History Study. Women were eligible if they were 13-21 years old, sexually active (5 years maximum), and had no history of cervical intraepithelial neoplasia, cervical procedures, or immunosuppression. At 4-month interval visits, we performed colpophotography to document the epithelium, HPV testing for 37 types by Roche Reverse Line Blot assay, and interviews to assess behaviours. This study selected women $(N=147)$ who had negative HPV results at the first two consecutive visits. Epithelial areas of interest were measured in the digitised colpophotographs by using computerised planimetry to produce pixel counts. The extent of ectopy was measured as a percentage of the total cervical face. Cox proportional hazards models examined ectopy as a predictor for incident HPV, defined as the first positive HPV result following the initial two negative results, adjusted for the number of new sexual partners. Outcomes included incidence of any HPV type; $\alpha-9$ HPV types (defined as 16, 31, 33, 35, 52, 58, 67); and a-3/15 HPV types (defined as 61, 71, 72, 81, 83, 84, 89).

Results The 147 women attended a total of 545 visits. The mean age at baseline was 17.2 years, mean age of menarche was 12.8 years, and mean age of first sex was 15.4 years. Self-reported race/ethnicity was 35 (24\%) Asian, 14 (10\%) African-American, 43 (29\%) Caucasian, 52 (35\%) Latina, and 3 (2\%) Other. The median ectopy measurement from the 545 visits was $14 \%$ (interquartile range $6-32 \%)$ of the total cervical face. Incident HPV of any type was found in $42(29 \%)$ women. The extent of ectopy was not significantly associated with incidence of any HPV type (HR 1.004 $p=0.63$ ); $\alpha-9$ HPV types (HR 0.99, $p=0.43$ ); or $\alpha-3 / 15$ HPV types (HR 1.02, $\mathrm{p}=0.18$ ). Results were unchanged when adjusted for new sexual partners in the past 8 months (HR 1.9, p<0.01)

Conclusions When measured quantitatively, the extent of cervical ectopy is not a risk factor for the acquisition of HPV infection in healthy adolescent women.

\section{3-S2.05 PREVALENCE OF TRICHOMONAS VAGINALIS AND CO- INFECTION WITH CHLAMYDIA TRACHOMATIS AND NEISSERIA GONORRHOEA IN THE USA AS DETERMINED BY THE APTIMA TRICHOMONAS VAGINALIS NUCLEIC ACID AMPLIFICATION ASSAY}

doi:10.1136/sextrans-2011-050109.113

${ }^{1} \mathrm{C}$ C Ginocchio, ${ }^{2} \mathrm{~K}$ Chapin, ${ }^{3} \mathrm{~J} S$ Smith, ${ }^{4} \mathrm{~J}$ Aslanzadeh, ${ }^{5} \mathrm{~J}$ Snook, ${ }^{5} \mathrm{C} S$ Hill, ${ }^{6} \mathrm{C}$ A Gaydos. ${ }^{1}$ North Shore-LIJ Health System Laboratories Lake Success, USA; ${ }^{2}$ Rhode Island Hospital Providence, USA; ${ }^{3}$ University of North Carolina, Chapel Hill, USA; ${ }^{4}$ Hartford Hospital, Clinical Laboratory Partners, Hartford, USA; ${ }^{5}$ Gen-Probe Incorporated, San Diego, USA; ${ }^{6} J o h n s$ Hopkins University, School of Medicine, Baltimore, USA

Background Trichomonas vaginalis (TV) is the most common curable sexually transmitted infection worldwide. True prevalence of TV infection is not well characterised as previous studies mainly used 